\title{
Therapeutic hypothermia for elevated intracranial pressure in traumatic brain injury: does it do more harm than good?
}

\author{
Kiwon Lee \\ Department of Neurosurgery and Neurology, The University of Texas Medical School at Houston, The Mischer \\ Neuroscience Institute, Memorial Hermann Texas Medical Center, Houston, USA
}

I have reviewed above article by Andrews et al. [1] with great interest. Reading this article has evidently produced me with a mixed feeling of both satisfying and unsatisfying emotional feedback for the reasons I have carefully outlined below.

This is yet another disappointment to the multisubspecialty medical and surgical communities, because now the concept and application of hypothermia, or perhaps better termed as targeted temperature modulation, seems to be failing in "neuro" applications. As many readers are likely aware of, Nielsen et al. [2] have reported a negative trial of comparing 33 degrees Celsius versus 36 degrees Celsius after cardiac arrest at the end of 2013. After questioning the true value of hypothermia in cardiac arrest victims, it is now being questioned as to whether applying hypothermia in traumatic brain injury (TBI) would confer any benefits in long term outcomes.

\section{The Reasons for Satisfying Feeling}

First, this trial was designed well not only from the pure statistical standpoint, but more importantly, it accurately reflected the current practice patterns of physicians and surgeons around the world regarding critical care for patients with TBI. For those with sustained, elevated intracranial pressure (ICP) defined in

Corresponding author: Kiwon Lee, M.D., FACP, FAHA, FCCM.

Department of Neurosurgery and Neurology, The University of Texas Medical School at Houston, The Mischer Neuroscience Institute, Memorial Hermann Texas Medical Center, Houston, 6431 Fannin St. Medical School Building 7.152, Houston, TX 77030, USA

Tel: 1-713-500-6128, Fax: 1-713-500-0665

E-mail: Kiwon.Lee@uth.tmc.edu

Korean J Anesthesiol 2015 December 68(6): 523-524 http://dx.doi.org/10.4097/kjae.2015.68.6.523 this study as greater than $20 \mathrm{mmHg}$ for at least 5 minutes, the step-by-step therapeutic approach was used which was appropriate. Instead of jumping right into the use of osmotic therapy such as mannitol and hypertonic saline, the study was designed to provide the essential, initial approach ("step 1") which was to provide elevating the head of the bed, mechanical ventilation and adequate sedation. It is nice to see that the authors have emphasized the importance of this critical stage and provided adequate sedation for all patients. If the study design did not include this critical step, the results would have been seriously confounded, even if it had sufficient statistical power.

Second, the trial acknowledged and adequately addressed many clinicians who believed in the value of early hypothermiacooling patients either prior to, or at the same time as, using osmotic therapy. There are neuroscience institutes in which patients with moderate to severe TBI would be cooled to 32-34 degrees Celsius from day 1 , moments after intubating the patients hoping for neuroprotection from the beginning of the injury. This study compared such therapeutic option with more conventional or standard therapy.

Third, this trial once again alarmed all clinicians about the potential harms that hypothermia may produce. The serious adverse events were more than three times that of the control group. The futility as well as safety concern called for early termination of the study. This is a lesson we've received in the past, but something that is worthwhile to reiterate.

\section{The Reasons for Unsatisfying Feeling}

First, while this study provided valuable, negative results for those who truly believed in early use of hypothermia, this study did not provide any valuable lessons for those who never really believed in "early" use of hypothermia. It is my opinion

(c) This is an open-access article distributed under the terms of the Creative Commons Attribution Non-Commercial License (http://creativecommons.org/ licenses/by-nc/4.0/), which permits unrestricted non-commercial use, distribution, and reproduction in any medium, provided the original work is properly cited. 
that hypothermia may be helpful in certain, specific population: refractory ICP crisis in patients with severe TBI, for instance. From an efficacy standpoint, there was a less than ideal selection of patients in this study. Simply randomizing all TBI patients with ICP greater than $20 \mathrm{mmHg}$ for 5 minutes after stage 1, may or may not represent those with truly severe TBI. Patients with mild to moderate injury may fit into the category of "high" ICP using above criteria. It is not surprising see that some of these patients would do well no matter what therapy is used. Preselected study population, therefore, may not be the right population for the value of hypothermia to be evident. As mentioned by the authors, hypothermia can decrease ICP in patients with TBI. What was not clear was whether successfully lowering ICP would lead to improved outcomes. This study showed that it did not. However, I would cautiously argue that the study might not have included the right patients, meaning, if hypothermia was used for those with refractory ICP crisis (defined by high ICP despite both step 1 and 2 applied), the outcomes might have improved.

Second, this study was not hypothermia plus standard therapy vs. standard therapy alone. If one reads the paper carefully, it is clear that the study was more like comparing hypothermia vs. mannitol and hypertonic saline (in the hypothermia group, mannitol and hypertonic saline were not given unless hypothermia failed to control the ICP). It was really cooling vs. standard therapy, NOT cooling plus standard vs. standard therapy alone. This is an important distinction because many clinicians do not use (or believe) cooling instead of standard therapy. Cooling for many of us is used in conjunction and does not replace our standard osmotherapy. Quite frankly, it is not surprising to observe no difference in long term outcome when comparing cooling vs. mannitol for elevated ICP for a cohort with mixed TBI severities. The motor GCS and $20 \mathrm{mmHg}$ ICP alone do not necessarily mean severe TBI. After reviewing the patient demographics, it is evident that the majority of patients in the both groups were a. moving their arms and legs and $b$. had bilateral pupil that were reactive to light. This signifies TBI of mixed population and not necessarily really sick group.
Third, there are many independent variables that may affect the long term neurological outcomes. Nutrition and advanced rehabilitation capabilities, for example, may affect the outcome rather significantly, independent of what the acute therapies might have been in the intensive care unit for the first few days of the injury. ICP as a single independent variable, cannot be used solely as an outcome predictor. Pressure in the brain is only one of many variables that contribute to the long term outcome. As such, controlling one variable is not likely to make a significant difference. This does not mean ICP is not an important variable in taking care of patients with moderate to severe TBI. In fact, it is very important. However, setting an arbitrary ICP value of greater than $20 \mathrm{mmHg}$ for 5 minutes alone without other pertinent information about the patient may not be sufficient to produce any significant impact on long term outcome. ICP of $22 \mathrm{mmHg}$ with cerebral perfusion pressure of $65 \mathrm{mmHg}$ may be tolerated just fine. ICP as an absolute value, and only value, does not determine long term outcome. It never did. Furthermore, numerous variables after being discharged from the hospital may play a role and it is extremely difficult to control these factors as a part of the investigational trial.

\section{Conclusion}

Hypothermia, when applied for a certain specific population at the discretion of treating physicians and surgeons, may produce benefits in maintaining adequate cerebral perfusion by lowering both metabolic demands and ICP while further potentially avoiding secondary neuronal injuries especially in severe injuries with refractory ICP crisis. Nonetheless, providing hypothermia instead of standard therapies such as mannitol and hypertonic saline in patients with mixed degrees of traumatic brain injury may not confer any long term outcome benefits. Furthermore, it is essential for clinicians to be reminded about the potential serious adverse effects that are associated with the use of hypothermia. It is easy to start hypothermia but not easy to maintain it without encountering serious adverse events that are not trivial.

\section{References}

1. Andrews PJ, Sinclair HL, Rodriguez A, Harris BA, Battison CG, Rhodes JK, et al. Hypothermia for Intracranial Hypertension after Traumatic Brain Injury. N Engl J Med 2015 [Epub ahead of print]. Available from http://dx.doi.org/10.1056/NEJMoa1507581

2. Nielsen N, Wetterslev J, Cronberg T, Erlinge D, Gasche Y, Hassager C, et al. Targeted temperature management at $33^{\circ} \mathrm{C}$ versus $36^{\circ} \mathrm{C}$ after cardiac arrest. N Engl J Med 2013; 369: 2197-206. 


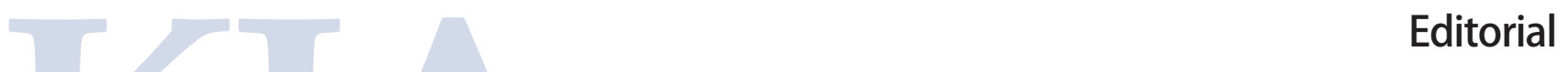 \\ Propofol as a controlled substance: poison or remedy
}

\author{
Young-Tae Jeon \\ Department of Anesthesiology and Pain Medicine, Seoul National University Bundang Hospital, Seongnam, Korea
}

Korea is the first, and perhaps only, country to classify propofol as a controlled substance. In February 2011, the Korean Ministry of Food and Drug Safety classified propofol as a psychotropic drug, making it illegal to prescribe or consume other than for stipulated treatments that may need sedation, such as a gastrointestinal endoscopy [1]. In this month's Korean Journal of Anesthesiology, we learned that Kim et al. [2] provided the reference data for the regulation of propofol in Korea as a controlled substance. The Korean Ministry of Food and Drug Safety considered the results of their survey.

In 2009, a report of nine cases of propofol abuse by medical personnel concluded that propofol required stricter control or designation as a controlled substance [3]. This study queried 95 councilors of Korean Society of Anesthesiologists on the abuse of propofol by their medical personnel. The nine cases included four anesthesiology residents, two other residents, an anesthesiology nurse, and two individuals with unknown backgrounds. Although this study has been criticized because the results are based on an incomplete survey of councilors whose recall may be inaccurate, it was the first report on propofol abuse in Korea.

Propofol has not been considered a controlled substance because it is not associated with physical dependency. Consequently, the addictive potential of the drug has received little attention. Although propofol has been used safely for over 30 years without significant addiction problems, the tragic death of pop icon Michael Jackson in 2009 brought the abuse of propofol by the general public into the spotlight. While physical dependency

Corresponding author: Young-Tae Jeon, M.D., Ph.D.

Department of Anesthesiology and Pain Medicine, Seoul National University Bundang Hospital, 82, Gumi-ro 173beon-gil, Bundang-gu, Seongnam 13620, Korea

Tel: 82-31-787-7493, Fax: 82-31-787-4063

E-mail: ytjeon@snubh.org

Korean J Anesthesiol 2015 December 68(6): 525-526

http://dx.doi.org/10.4097/kjae.2015.68.6.525 is rare, psychological dependency is an important phenomenon. Euphoria, sexual fantasies and dreams, and sexual disinhibition upon awakening are well-known effects of propofol and can also occur after anesthesia and monitored anesthesia care.

Many countries agree that propofol should be subject to stricter control. However, misuse is not a real problem in those countries. They are concerned that stricter control of propofol would involve an increased administrative load. The US Food and Drug Administration requires that the drug be administered by healthcare professionals trained in the administration of general anesthesia. In the UK, it is always administered by anesthetists or intensivists. In the past, addiction has been a major issue for anesthesia personnel [4]. It has been suggested that a major contributor to propofol addiction is easy access. Although access alone does not result in drug abuse, tighter control allows for earlier detection and documentation in suspected cases of abuse [4].

What makes circumstances in Korea different from those in other countries? The authors argue that propofol abuse in nonhealthcare professionals is not a social issue and more serious substance abuse including narcotics and marijuana is the main concern of regulatory body in other countries. Unlike the general situation in most countries, non-healthcare professional are exposed to propofol abuse in Korea. Most cases enrolled in this study were non-healthcare professional single women in their 20s. These cases became social issues. We hope that this study is a lesson for other countries where propofol abuse occurs. Paracelsus' famous quotation may be modified for this century as "Poison is in everything, and no thing is without poison. The usage (instead of dosage) makes it either a poison or a remedy."

(c) This is an open-access article distributed under the terms of the Creative Commons Attribution Non-Commercial License (http://creativecommons.org/ licenses/by-nc/4.0/), which permits unrestricted non-commercial use, distribution, and reproduction in any medium, provided the original work is properly cited. 


\section{References}

1. Psychotic drugs control act. Act No. 5529, 28 Feb 1998. Available from http://www.moleg.go.kr/lawinfo/engLawInfo;jsessionid=RCuRlvvr9 hpcP1sHpsKfkdWmDc8d1DeMgUrwutQo4npebIeTevh3XWVD3t0Wdm4s.moleg_a1_servlet_engine2?pstSeq=58352\&pageIndex=4.

2. Kim E, Kim S, Hyun Y, Noh Y, Jung H, Han S, et al. Clinical and psychological characteristics of propofol abusers in Korea: a survey of propofol abuse in 38, non-healthcare professionals. Korean J Anesthesiol 2015; 68: 586-93.

3. Lee S, Lee MS, Kim YA, Ahn W, Lee HC. Propofol abuse of the medical personnel in operation room in Korea. Korean J Leg Med 2010; 34: 101-7.

4. Silverstein JH, Silva DA, Iberti TJ. Opioid addiction in anesthesiology. Anesthesiology 1993; 79: 354-75. 